\title{
Morbilidad hospitalaria en pacientes con infección por VIH
}

\author{
I. M. ARIAS MIRANDA, M. E. GONZÁLEZ GARCÍA ${ }^{1}$, M. L. GARCÍA-ALCALDE \\ FERNÁNDEZ ${ }^{2}$, B. DE LA FUENTE GARCÍA ${ }^{2}$, M. T. CAMPOAMOR SERRANO ${ }^{2}$, \\ J. MORÍS DE LA TASSA ${ }^{3}$
}

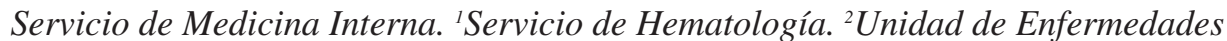
Infecciosas. ${ }^{3}$ Servicio de Medicina Interna. Hospital de Cabueñes. Gijón. Departamento de Medicina. Universidad de Oviedo

\author{
HOSPITAL MORBIDITY IN HIV INFECTED PATIENTS
}

\section{RESUMEN}

Fundamento y objetivo: Con el aumento de la esperanza de vida en pacientes con infección por el virus de la inmunodeficiencia humana (VIH) tras el tratamiento antirretroviral de gran actividad (TARGA), las causas de hospitalización han cambiado. Predominan ahora comorbilidades como la hepatopatía crónica, la patología cardiovascular o los efectos secundarios de los tratamientos. Nuestro objetivo es determinar las características clinicoepidemiológicas actuales de estos pacientes.

Material y métodos: Estudio descriptivo y retrospectivo. Revisamos las historias clínicas de los ingresos de pacientes con infección por el VIH hospitalizados en los servicios médicos de nuestro hospital en el transcurso de un año.

Resultados: Hubo 125 ingresos de 82 pacientes, un $77 \%$ varones y un $71 \%$ con infección por el virus de la hepatitis C (VHC). La edad media fue de 42 años. En el 64\% la vía de transmisión fue la drogadicción parenteral. Un 36\% recibían TARGA y un 51\% reunían criterios sida. Los motivos de ingreso más frecuentes fueron la patologia respiratoria $(32 \%)$ y las enfermedades definitorias de sida (EDS) con un $22 \%$, de las que la tuberculosis (TBC) fue la más frecuente. Un $10 \%$ fueron ingresados en la Unidad de Cuidados Intensivos (UCI). La mortalidad fue del $11 \%$ pacientes/año.

Conclusiones: La mayoría de los pacientes fueron varones, con coinfección por el VHC y usuarios de drogas vía parenteral, siendo las causas de ingreso más frecuentes las patologías respiratorias y las enfermedades oportunistas. La TBC permanece siendo la EDS más frecuente.

PALABRAS CLAVE: Hospitalización. VIH. Sida.

\section{ABSTRACT}

Background and objetive: With the increasing life expectancy in human immunodeficiency virus (HIV) infected patients since the highly active antiretroviral therapy (HAART), other underlying comorbilities such as chronic liver patology, cardiovascular diseases or side effects of treatment may lead to hospitalization. The purpose of this study is to determine the clinical and epidemiological characteristics of these patients.

Material and methods: A descriptive-retrospective study. We reviewed the clinical records of patients with HIV infection admitted in our medical departments through a year.

Results: There were 125 hospitalizations of 82 patients. $77 \%$ were males and $71 \%$ had hepatitis C virus (HCV) infection. The median age was 42 years. $64 \%$ HIV infection had been acquired by sharing material for intravenous drug use. A $36 \%$ were receiving HAART and $51 \%$ had AIDS. The respiratory tract patology $(32 \%)$ and AIDS-defining illnesses (22\%) were the most frequent causes of hospitalization, and the main opportunistic disease was tuberculosis (TBC). Ten per cent were admitted in the Intensive Care Unit. Mortality rate was $11 \%$ patients/year.

Conclusions: Most of the patients were male, drug users and $\mathrm{HCV}$ positive, and the admissions to hospital were mostly due to respiratory patology and opportunistic diseases. TBC remains like the most frequent AIDS-defining illness.

KEY WORDS: Hospitalization. HIV. AIDS. Tassa J. Morbilidad hospitalaria en pacientes con infección por VIH. An Med Interna (Madrid) 2006; 23: 519-524.

\section{INTRODUCCIÓN}

En 1996 tras autorizarse el primer inhibidor de la proteasa se inició la transformación del esquema terapéutico para la infección del virus de la inmunodeficiencia humana (VIH). En los meses siguientes se extendió su uso produciendo la mejora de los pacientes reflejándolo también a nivel económico ya que, a pesar del gran coste de ese tipo de medicación, se compensó por la disminución en el uso de los servicios hospi- talarios (1,2). El tratamiento antirretroviral de gran actividad (TARGA) ha prolongado la supervivencia y ha inducido grandes efectos en los pacientes con infección por el VIH, observándose en los últimos años un cambio en el espectro de sus manifestaciones (3-5). Es difícil estimar las características exactas de estos cambios si partimos de hallazgos publicados en trabajos de fuera de nuestro país, ya que nuestra población tiene particularidades que influyen en la evolución de la enfermedad. Así podemos ver en nuestro entorno una mayor tasa

Trabajo aceptado: 13 de junio de 2006 
de portadores del virus de la hepatitis C (VHC) secundaria a la alta prevalencia de drogadicción en estos pacientes, que se suele acompañar a su vez de una situación social desfavorable (6-8). A partir del espectro de enfermedades que ocasionan los ingresos se puede deducir la tendencia de la historia natural de la infección (9-15). Es por esto que nuestro objetivo es describir algunas características clinicoepidemiológicas de estos ingresos y con ello conocer mejor a nuestros pacientes para poder diseñar en el futuro mejores tácticas de manejo.

\section{MATERIAL Y MÉTODOS}

\section{CRITERIOS DE INCLUSIÓN}

Presentamos un estudio descriptivo y retrospectivo efectuado en el Hospital de Cabueñes, centro hospitalario de la Red Pública del Servicio de Salud del Principado de Asturias (SESPA). Atiende a la población del Área Sanitaria V con un número aproximado de 300.000 habitantes. La selección de los pacientes fue mediante la búsqueda a través de los informes de alta de los servicios médicos, desde el 1 de enero hasta el 31 de diciembre de 2004, de pacientes mayores de 14 años diagnosticados de infección por VIH, con y sin diagnóstico de sida.

\section{VARIABLES ANALIZADAS}

Se recogieron datos de sexo, edad, infección por $\mathrm{VHC}$, tiempo conocido de infección por el VIH, existencia de problema social, situación inmunológica objetivada por número de linfocitos CD4+, enfermedad definitoria de sida (EDS), diagnóstico principal al alta, ingreso en la Unidad de Cuidados Intensivos (UCI), fallecimientos y tratamiento que efectuaban en el momento del ingreso, pacientes sin tratamiento o con TARGA. Dada la importancia de los problemas sociales para el seguimiento y adherencia al tratamiento se ha definido una variable que permite conocer la situación social, que incluiría trastornos psiquiátricos, de toxicodependencia, inestabilidad sociolaboral, ausencia de apoyo familiar, de domicilio fijo (indigentes) o desarraigo social.

Se consideraron diagnósticos tanto los procesos confirmados como los presuntivos. Se consideraron episodios clínicos definitorios de sida los incluidos en la categoría C de la clasificación de los Centers for Disease Control (CDC) de 1993 (16).

\section{ANÁLISIS ESTADÍSTICO}

Se realizó un estudio descriptivo de las variables analizadas mediante proporciones para las variables cualitativas y la media para las variables cuantitativas. Se consideró cada uno de los ingresos de forma independiente y en todos los cálculos se consideró como denominador el número total de ingresos/año. El análisis estadístico se realizó mediante el programa SPSS (Statistical Package for Social Sciences) para Windows, versión 12.0 con licencia de uso. Se han aplicado la prueba de la $\chi^{2}$ para la comparación de proporciones. Se consideraron valores significativos valores de $\mathrm{p}$ inferiores a 0,05 .

\section{RESULTADOS}

En el 2004 hubo un total de 19.140 altas en nuestro hospital, de ellas 125 se produjeron en 82 pacientes con infección por VIH en los servicios médicos (Tabla I). La edad media de los pacientes en el momento del ingreso fue de 42 años, con un tiempo medio conocido de infección por el VIH de 8,6 años. Un 36\% estaban siendo tratados con TARGA en el momento de la hospitalización; no encontramos diferencias significativas frente a los que no estaban con TARGA a la hora de ingresar por enfermedades definitorias de sida (EDS) o respecto a la mortalidad. De los 125 ingresos 77,6\% eran hombres y el $22,4 \%$ mujeres. El $64 \%$ de los ingresos se trataban de pacientes que eran o habían sido consumidores de drogas inyectadas que fue la vía de transmisión más frecuente. La transmisión vía sexual se recogió en el $27 \%$ de los ingresos (19\% heterosexual y $8 \%$ homosexual) y por hemoderivados en un $2 \%$.

Se determinó la presencia de problema social en un $41 \%$ de los pacientes. Asimismo encontramos una asociación con significación estadística $(\mathrm{p}=0,017)$ de los pacientes adictos a droga vía parenteral (ADVP) y una mala situación social ocasionada por las características de este grupo de población, no encontrando diferencias significativas entre el grupo de ADVP y la mortalidad.

$\mathrm{El} 71 \%$ de los ingresos presentaban anticuerpos frente al VHC, hecho que se relaciona directamente con el método de adquisición del VIH en los ADVP $(\mathrm{p}=0,00)$. La proporción de ingresos de pacientes con sida fue de un $51 \%$. El recuento

\section{TABLA I}

\section{CARACTERÍSTICAS DE LOS INGRESOS}

Total de ingresos 125

$\mathrm{N}^{\circ}$ de pacientes

Edad media (años)

42,6

$$
\begin{aligned}
& \text { Sexo } \\
& \text {-Hombres } \\
& \text {-Mujeres }
\end{aligned}
$$

Tiempo medio conocido de infección por VIH (años)

Pacientes con infección por VHC

Mecanismo de adquisición de la infección

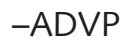

-Heterosexual

$19 \%$

-Homosexual

$8 \%$

-Desconocido

-Hemoderivados

$5 \%$

$4 \%$

Sida

$51 \%$

Mortalidad global

$11 \%$

Problema social

Con TARGA en el momento del ingreso $41,6 \%$

CD4+ $\leq 200 / \mu l$

Ingresos en $\mathrm{UCl}$

$36 \%$

$36 \%$

$10 \%$ 
medio de células CD4+ fue de 306 y un 36\% del total de los ingresos tenían una cifra de CD4+ menor de 200 células/ml. El $22 \%$ de las hospitalizaciones fueron motivadas por EDS como causa principal de ingreso (Fig. 1), objetivándose niveles de CD4 inferiores en este grupo de pacientes $(p=0,001)$. La infección tuberculosa fue la EDS más frecuente entre los pacientes estudiados (15 de 82 pacientes) siendo motivo de ingreso de manera directa en 7 ocasiones, seguido de la neumonía por $P$. jiroveci y la leucoencefalopatía multifocal progresiva (Fig. 2). En relación a otros diagnósticos no definitorios de sida categorizados en grandes grupos de patologías (Tabla II), encontramos como motivo de ingreso más frecuente la patología respiratoria con un $32 \%$, (con neumonía bacteriana 27 ingresos y con agudización de la enfermedad pulmonar obstructiva crónica (EPOC) 10). Con frecuencias menores están las EDS con un $22 \%$ y con un $15 \%$ las enfermedades digestivas $(6 \%$ por descompensación de hepatopatía por VHC).
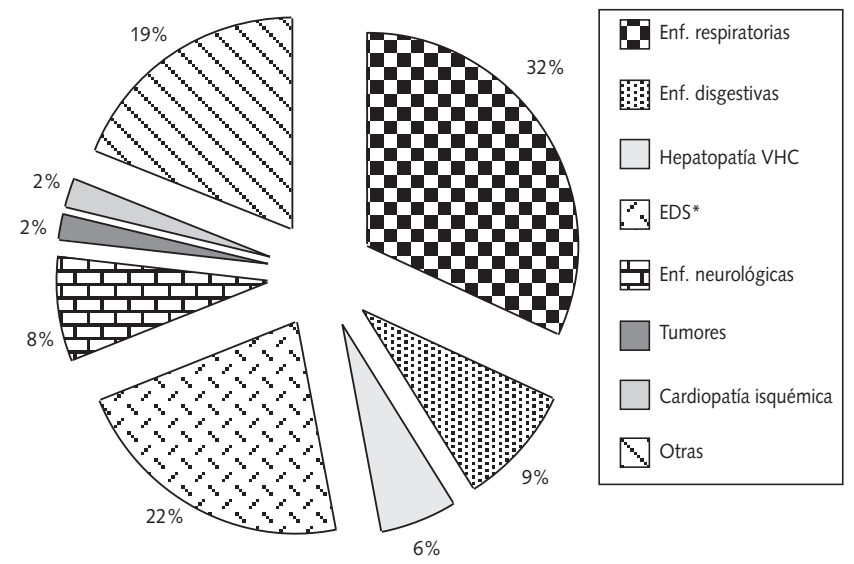

Fig. 1. Causas de ingreso.

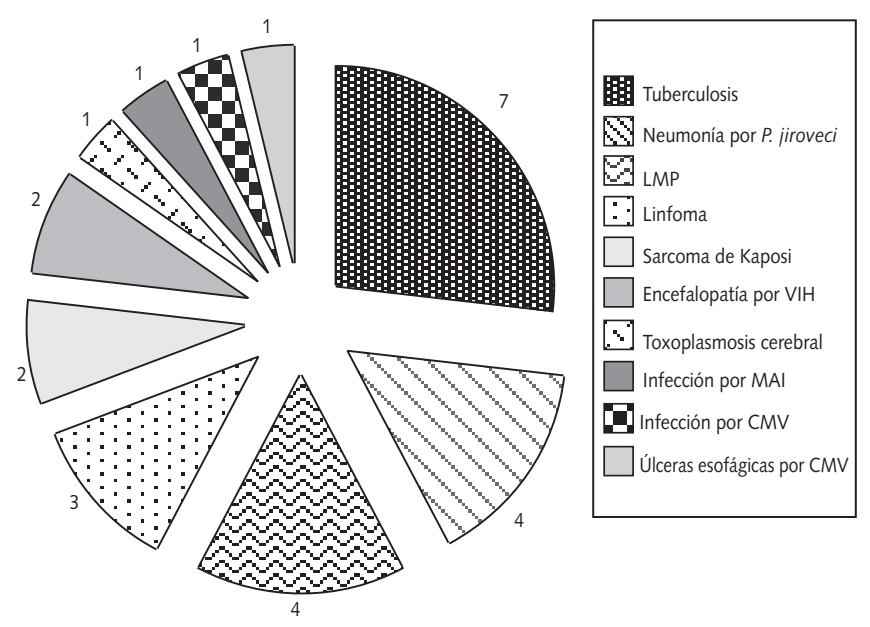

Fig. 2. EDS* como motivo de ingreso.

MAY: Mycrobacterium Avium Intracellulare; CMV: Citomegalovirus; LMP: Leucoencefalopatía multifocal progresiva.

En nuestra serie 13 pacientes, un $10 \%$ de los ingresos, se trasladaron a la UCI, 5 de ellos presentaban criterios de sida. Las causas más frecuentes fueron las infecciosas como la neumonía con 3 ocasiones ( 2 bacterianas y una por $P$. jiroveci) y la
TABLA II

DIAGNÓSTICOS PRINCIPALES DE INGRESO. NO EDS

Enfermedades respiratorias

-Neumonía bacteriana

-EPOC agudizado

- Infección respiratoria vías altas

(traqueobronquitis, sinusitis, bronquitis) 6

-Otras enfermedades digestivas

Total

2

39

Enfermedades digestivas

-Descompensación hepatopatía VHC 8

-Otras enfermedades digestivas 11

Total

19

Enfermedades neurológicas

-Meningitis

3

-Crisis comicial

2

-Lesiones ocupantes de espacio cerebrales 2

-Hidrocefalia normotensiva

-Cefalea

-Enfermedad de Parkinson

Total

1

1

1

10

Enfermedades cardiovasculares

-Enfermedad coronaria

3

Enfermedades endocrinas

Tumores

-Hepatocarcinoma

- Adenocarcinoma pulmonar

-Adenocarcinoma de mama Total

1

1

Otras Infecciones

-Abscesos

-Artritis infecciosa

2

Intoxicaciones medicamentosas 2

Síndrome febril de origen no filiado 4

Síndrome depresivo 1

Acidosis láctica por TARGA 2

Otras

meningitis con 2 (una meningocócica y otra neumocócica) (Tabla III). Todos evolucionaron favorablemente. No se encontramos diferencias significativas entre recibir TARGA o tener criterios de sida para ingresar en la UCI.

En el periodo estudiado fallecieron 14 pacientes que representan un $11 \%$ de los ingresos. La media de células CD4 de éstos fue inferior respecto a los sobrevivientes. No se obtuvo diferencia estadística entre los pacientes con TARGA. Sí observamos que los pacientes portadores del VHC no presentaron mayor mortalidad $(\mathrm{p}=0,013)$, destacando únicamente un fallecimiento por hepatocarcinoma en un paciente con previa hepatopatía por VHC. Las causas más frecuentes de muerte fueron la tuberculosis, la neumonía y el sarcoma de Kaposi (Tabla IV). 
TABLA III

CAUSAS DE INGRESO EN UCI

\begin{tabular}{ll}
\hline Neumonía & 3 \\
Meningitis & 2 \\
Cardiopatía isquémica & 2 \\
Intoxicación medicamentosa & 1 \\
Hepatopatía VHC & 1 \\
Pleuropericarditis aguda & 1 \\
Cetoacidosis diabética & 1 \\
Toxoplasmosis cerebral & 1 \\
Acidosis láctica por tratamiento & 1 \\
$\quad$ antirretroviral & 13 \\
\hline
\end{tabular}

VHC: Virus de la hepatitis C.

\section{TABLA IV}

CAUSAS DE FALLECIMIENTO

\begin{tabular}{lc}
\hline Diagnóstico principal & Pacientes \\
\hline Sarcoma de Kaposi & 2 \\
Neumonia & 2 \\
Tuberculosis & 2 \\
Hepatocarcinoma & 1 \\
LMP & 1 \\
Encefalopatía por VIH & 1 \\
Adenocarcinoma de mama & 1 \\
Linfoma cerebral & 1 \\
Lesiones ocupantes de espacio cerebrales & 1 \\
Meningitis & 1 \\
Infección diseminada por MAI & 1 \\
Fallecidos en total & 14
\end{tabular}

MAI: Mycobacterium Avium Intracelullare.

LMP: Leucoencefalopatía multifocal progresiva.

\section{DISCUSIÓN}

Como en el sur de Europa en nuestros pacientes predominan los ADVP lo que explica en gran medida la elevada prevalencia de anticuerpos frente al VHC que es la coinfección crónica activa más prevalente en esta población (17-22). Klein y cols. demostraron que éstos tienen el doble de riesgo de ingreso hospitalario que aquellos sin infección por VHC (18). Nosotros encontramos que aproximadamente tres cuartas partes de los ingresos presentaban esta coinfección, proporción cercana a otras series donde llegan al 86\% (19). Esto justificaría el plantear tratamiento de esta infección como punto crucial para mejorar el pronóstico de estos pacientes. Aunque es conocido que los pacientes con coinfección por VHC tienen mayor morbilidad nosotros no encontramos diferencia estadísticamente significativa a la hora de evaluar si ingresaban más por una EDS.
La infección por VIH favorece el desarrollo a TBC activa y las recurrencias, también a su vez la TBC puede acelerar la progresión de la infección por el VIH incrementando la replicación viral lo que se acompaña de una mayor mortalidad (23). En nuestra serie es la causa más frecuente de EDS entre los pacientes estudiados y fue la causa responsable de su ingreso en 7 casos. De ellos 2 fueron tuberculosis pulmonar y 5 extrapulmonar de acuerdo con la mayor prevalencia de las formas extrapulmonares en pacientes con infección por VIH.

Como en otras series las infecciones respiratorias, sobre todo las neumonías, representan la mayoría de los motivos de ingresos en los últimos tiempos. Nuestro estudio también confirma dicha tendencia siendo más frecuentes las neumonías bacterianas y la agudización de la EPOC (6). Encontramos pocos casos de neumonía por $P$. jiroveci, en relación a otros estudios publicados, debido al uso extendido de la profilaxis y a la disminución de los casos de inmunodepresión severa, lo que sugiere un previsible aumento de episodios de neumonía bacteriana en un futuro (24-26).

Los pacientes con infección por VIH tienen un riesgo incrementado de desarrollar cáncer respecto a la población general, de hecho el comienzo de la epidemia del sida en 1981 se detectó ante el aumento en la incidencia de una rara neoplasia como el sarcoma de Kaposi (27). En 1982 los U.S. Centers for Disease Control and Prevention (CDC) propusieron la primera definición de sida incluyendo como neoplasias definitorias de sida el sarcoma de Kaposi y el linfoma primario del sistema nervioso central, posteriormente se añadieron el linfoma no Hodgkin y el cáncer de cérvix invasivo (28-31). En nuestra serie predomina el linfoma como tumor definitorio de sida en 3 ingresos seguido del sarcoma de Kaposi con 2. Las neoplasias fueron responsables de 5 fallecimienots (dos sarcomas de Kaposi, un linfoma, un adenocarcinoma de mama y un hepatocarcinoma). Otro tumor que se prevé su aumento es el hepatocarcinoma; ésto se explicaría por las características de esta población con alta prevalencia de infección crónica por VHC, asociado o no al abuso de alcohol, junto al aumento en la esperanza de vida (32).

Estos enfermos también presentan un elevado riesgo de enfermedad coronaria prematura. La mayoría de los pacientes descritos en los estudios tenían una grave dislipemia atribuída al uso de inhibidores de la proteasa. Aunque la hiperlipidemia y la resistencia a la insulina asociada a la utilización de inhibidores de la proteasa pueden facilitar el desarrollo de esta patología de manera precoz, parece que la arterioesclerosis prematura no está producida por los efectos metabólicos del tratamiento teniendo mayor influencia una carga viral elevada $(33,34)$. En nuestra experiencia vemos que 3 de los ingresos en el último año se deben a cardiopatía isquémica en pacientes menores de 60 años, 2 de ellos con TARGA. Todos tuvieron una buena evolución de su cardiopatía sin determinarse ninguna complicación durante su estancia hospitalaria. Estos hallazgos concuerdan con otros donde se observa que estos pacientes sufren infartos de miocardio a una edad joven y presentan una buena evolución en el curso del ingreso.

También consideramos interesante estudiar el número de traslados a la UCI en estos pacientes. Según Nuesh y cols. las admisiones a una UCI han aumentado significativamente en los últimos años, exactamente de un 6,3 a un $11,8 \%$ (2). En comparación con Escolano y cols., que describen 8 pacientes tratados en una UCI de un total de 1.343 ingresos hospitalarios, encontramos en nuestra serie una elevada tasa de ingre- 
sos en UCI ya que de 125 ingresos 14 se trasladaron a UCI (11\%), evolucionando todos favorablemente (15). Se han descrito como primeras causas de ingreso en estas unidades los cuadros sépticos (hasta un 75\%), siendo el origen pulmonar el más frecuente (35).

Finalmente vemos que la enfermedad por VIH se presenta de manera muy diferente respecto a cuando se describió por primera vez hace dos décadas, al menos en los países desarrollados donde el TARGA está disponible. No cabe duda que el fracaso terapéutico es un factor crucial que contribuye a una mayor morbimortalidad (36-39). Esto ocurre principalmente por resistencia virológica, mal cumplimiento por falta de adherencia terapéutica y en grupos desfavorecidos (bajo estrato social, patologías psiquiátricas, indigentes...) (40-44). De acuerdo a nuestros datos un $41 \%$ de los ingresos presentaban problemas sociales, circunstancia que sin duda determina la evolución de su enfermedad.

Por último comentar las limitaciones de nuestro estudio, ya que al ser una recogida de información retrospectiva una de las desventajas es la heterogeneicidad de los datos que limita una correcta evaluación. También es difícil extraer conclusiones respecto a la morbilidad y mortalidad debido al reducido número de casos, por lo que se necesitaría un tamaño de muestra mucho mayor para poder obtener potencia estadística. Otros inconvenientes son el corto periodo de tiempo estudiado que impide la posibilidad de comparar y observar la evolución respecto a años previos, y el analizar únicamente las enfermedades que requirieron ingreso hospitalario no representando patologías que fueron manejadas ambulatoriamente.

\section{Bibliografía}

1. Bozzette SA, Joyce G, McCaffrey DF, Leibowitz AA, Morton SC, Berry SH, et al. The HIV Cost and Services Utilization Study Consortium. Expenditures for the Care of HIV-Infected Patients in the Era of Highly Active Antiretroviral Therapy. N Engl J Med 2001; 344: 817823

2. Palella FJ, Delaney KM, Moorman AC et al. Declining morbidity and mortality among patients with advanced human immunodeficiency virus infection. N Engl J Med 1998; 338: 853-860.

3. Paul S, Gilbert HM, Ziecheck W, Jacobs J, Sepkowitz KA. The impact of potent antiretroviral therapy on the characteristics of hospitalized patients with HIV infection. AIDS 1999; 13: 415-418.

4. San Andrés Rebollo FJ, Rubio García R, Castilla Catalán J, Pulido Ortega F, Palao G, De Pedro Andrés I, et al. Estudio de la mortalidad y supervivencia en una cohorte de 1.115 pacientes con infección VIH (1989-97). An Med Interna (Madrid) 2004; 11: 523-532.

5. Alonso Socas MM, Gómez Sirvent JL, Santolaria Fernández F, Rodríguez Moreno F, Essardas H, Rodríguez Rodríguez E, et al. Eficacia del tratamiento antirretroviral en pacientes con infección por el virus de la inmunodeficiencia huamana. Estudio de 807 pacientes. Med Clin (Barc) 2000; 115: 481-486.

6. Rubio Rivas C, Egido García R, Nogues Biau A. Hepatitis C crónica en pacientes VIH coinfectados. Estudio de 55 casos con biopsia hepática. Med Clin (Barc) 2004; 123: 441-444.

7. Tural C, Clotet B. La era del tratamiento antirretroviral de gran actividad... y de la coinfección por el virus de la hepatitis C. Med Clin (Barc) 2004; 123: 460-462.

8. Sánchez-Quijano A, Leal M, Lissen E. Hepatitis crónica por virus C en pacientes coinfectados por el virus de la inmunodeficiencia humana. Gastroenterol Hepatol 2002; 25: 423-31.

9. Fernández-Rivera J, Macías J, García-García JA, Ramos AJ, Leal M, Pineda JA. Efecto del tratamiento antirretroviral de gran actividad sobre la forma de presentación de sida definido por episodios clínicos. Med Clin (Barc) 2002; 118: 686-688.

10. Perbost I, Malafronte B, Pradier C, Santo LD, Dunais B, Counillon E, et al. In the era of highly active antiretroviral therapy, why are HIV-infected patients still admitted to hospital for an inaugural opportunistic infection? HIV Med 2005; 6: 232-239.

11. Iribarren JA, González-García J. Ingresos hospitalarios y mortalidad en pacientes con sida en la era TARGA: ¿hacia dónde vamos? Enferm Infecc Microbiol Clin 2004; 22: 129-132.

12. Castilla V, Alberdi JC, Barros C, Gómez J, Gaspar G, Sanz J. Cohorte multicéntrica de pacientes con infección VIH de la corona metropolitana sudeste de Madrid (COMESEM): fundamentos, organización y resultados iniciales. Rev Clin Esp 2003; 203: 170-177.

13. Teira R, Suárez-Lozano I, Muñoz P, Viciana P, Lozano F, Galindo MJ, et al. Patrón epidemiológico de la infección por el VIH en 15 hospitales españoles: casos de nuevo diagnóstico incluidos en la cohorte VACH en 2001 y 2002. Enferm Infecc Microbiol Clin 2005; 23: 213-217.

14. Valencia ME, Martín-Carbonero L, López M, Soriano V, Moreno V González Lahoz J. Motivo de ingreso hospitalario en pacientes con infección por el virus de la inmunodeficiencia humana en la era del tratamiento antirretroviral de gran actividad. Med Clin (Barc) 2002; 119: 293-295.

15. Escolano Hortelano CM, Ramos Rincón JM, Gutiérrez Rodero F, Masia Canuto M, Hernández Aguado I, Benito Santaleocadia C, et al. Cambios en el espectro de la morbilidad y la mortalidad en los ingresos hospitalarios de los pacientes con infección por el virus de la inmunodeficiencia humana durante la era del tratamiento antirretroviral de gran actividad. Med Clin (Barc) 2004; 122: 1-5.

16. CDC. 1993 Revised classification system for HIV infection and expanded surveillance case definition for AIDS among adolescent and adults. MMWR 1992; 41: 1-19.

17. González-García JJ, Mahillo B, Hernández S, Pacheco R, Diz S, García $\mathrm{P}$, et al. Estudio multicéntrico sobre prevalencia de las coinfecciones por virus de la hepatitis, indicación de tratamiento de hepatitis crónica C y necesidad de transplante hepático en pacientes infectados por el VIH en España. Estudio GESIDA 29/02-FIPSE 12185/01. Enferm Infecc Microbiol Clin 2005; 23: 340-348.

18. Klein MB, Lalonde RG, Suissa S. The impact of hepatitis $\mathrm{C}$ virus coinfection on HIV progression before and after highly active antiretroviral therapy. J Acquir Immune Defic Syndr 2003; 33: 365-372.

19. Rodríguez-Vidigal FF, Habernau A. Motivo de ingreso en pacientes infectados por el virus de la inmunodeficiencia humana en un área rural. Papel de la hepatopatía crónica. Enferm Infecc Microbiol Clin 2004; 22 : 138-41.

20. Bica I, McGovern B. Dhar R, Stone D, McGowan K, Sheib R, et al. Increasing mortality due to end-stage liver disease in patients with human inmunodeficiency virus infection. Clin Infect Dis 2001; 32: 492 497.

21. Monga HK, Rodríguez-Barradas MC, Breaux K, Khattak K, Troisi CL, Velez $\mathrm{M}$, et al. Hepatitis $\mathrm{C}$ infection-related morbility and mortality among patients with human inmunodeficiency virus infection. Clin Infect Dis 2001; 33: 240-247.

22. Kim WR, Gross JB, Poterucha JJ, Locke GR, Dickson ER. Outcome of hospital care of liver disease associated with hepatitis $\mathrm{C}$ in United States. Hepatology 2001; 33: 201-206.

23. Hung CC, Chen MY, Hsiao CF, Hsieh SM, Sheng WH, Chang SC. Improved outcomes of HIV-1-infected adults with tuberculosis in the era of highly active antiretroviral therapy. AIDS 2003; 17: 2615-2622.

24. Chernilo S, Trujillo S, Kahn M, Paredes M, Echevarría G, Sepúlveda C. Enfermedades pulmonares en pacientes infectados con VIH hospitalizados en el Instituto Nacional del Tórax. Rev Méd Chile 2005; 133: $517-$ 524.

25. Wolff AJ, O'Donnell AE. Pulmonary manifestations of HIV infection in the era of highly active antiretroviral therapy. Chest 2001; 120: 18881893 
26. Dufour V, Cadranel J, Wislez M, Lavole A, Bergot E, Parrot A, et al. Changes in the pattern of respiratory diseases necessitating hospitalization of HIV-infected patients since the advent of highly active antiretroviral therapy. Lung 2004; 182: 331-341.

27. Cheung MC, Pantanowitz L, Dezube BJ. AIDS-Related malignancies: Emerging challenges in the era of highly active antiretroviral therapy. Oncologist 2005; 10: 412-426.

28. CDC. 1993 revised classification system for HIV infection and expanded surveillance case definition for AIDS among adolescent and adults. Morbidity Mortality Weekly Report 1992; 41: 961-962.

29. Cattelan AM, Calabro ML, De Rossi A, Aversa SM, Barbierato M, Trevenzoli M, et al. Long-term clinical outcome of AIDS-related Kaposi's sarcoma during highly active antiretroviral therapy. Int J Oncol 2005; 27: 779-785.

30. Bonnet F, Lewden C, May T, et al. Malignancy-related causes of death in human immunodeficiency virus-infected patients in the era of highly active antiretroviral therapy. Cancer 2004; 101: 317-324.

31. Louie JK, Hsu LC, Osmond DH, et al. Trends in causes of death among persons with acquired immunodeficiency syndrome in the era of highly active antiretroviral therapy, San Francisco, 1994-1998. J Infect Dis 2002; 186: 1023-1027.

32. Murillas J, Del Río M, Riera M, Vaquer P, Salas A, Leyes M, et al. Increased incidence of hepatocellular carcinoma (HCC) in HIV-1 infected patients. Eur J Intern Med 2005; 16: 113-115.

33. Glesby MJ. Coronary Heart Disease in HIV-infected Patients. Curr HIV/AIDS Rep 2005; 2: 68-73.

34. Matetzky S, Domingo M, Kar S, Noc M, Shah PK, Kaul S, et al. Acute myocardial infarction in human immunodeficiency virus-infected patients. Arch Intern Med 2003; 16: 457-460.

35. Casalino, E, Wolff, M, Ravaud, P, Choquet, C, Bruneel, F, Regnier, B.
Impact of HAART advent on admission patterns and survival in HIVinfected patients admitted to an intensive care unit. AIDS 2004; 18: 1429-1433.

36. Bonnet F, Lewden C, May T, Heripret L, Jougla E, Bevilacqua S, et al. Opportunistic infections as causes of death in HIV-infected patients in the HAART era in France. Scand J Infect Dis 2005; 37: 482-487.

37. Manavi K, McMillan A. A significant proportion of HIV-infected patients admitted to hospital have immunosuppression as a result of failure of highly active antiretroviral therapy. HIV Med 2004; 5: 360-363.

38. Martín-Sánchez V, Ortega-Valin L, Pérez-Simón Mdel R, Mostaza-Fernández JL, Ortiz de Urbina-González JJ, Rodríguez-María M, et al; Grupo de Trabajo sobre Adhesion a TARGA en la provincia de Leon. Factors predicting lack of adherence to highly active antiretroviral treatment. Enferm Infecc Microbiol Clin 2002; 20: 491-497.

39. Nuesch R, Geigy N, Schaedler E, Battegay M. Effect of highly active antiretroviral therapy on hospitalization characteristics of HIV-infected patients. Eur J Clin Microbiol Infect Dis 2002; 21: 684-687.

40. Mocroft A, Monforte A, Kirk O, Johnson MA, Friis-Moller N, Banhegyi D, et al. EuroSIDA study group. Changes in hospital admissions across Europe: 1995-2003. Results from the EuroSIDA study. HIV Med 2004; 5: 437-47.

41. Altes J, Guadarrama M, Force L et al. The impact of highly active antiretroviral therapy on HIV-related hospitalisations in 17 county hospitals in Catalonia, Spain. AIDS 1999; 13: 1418-1419.

42. Beck EJ, Mandalia S, Williams I et al. Decreased morbidity and use of hospital services in English HIV-infected individuals with increased uptake of antiretroviral therapy 1996-1997. AIDS 1999; 13: 2157-2164.

43. Mocroft A, Barry S, Sabin CA, et al. The changing pattern of hospital admissions to a London Hospital of patients with HIV: 1988-1997. AIDS 1999; 13: 1255-1263. 